\title{
Energy harvested end nodes and performance improvement of LoRa networks
}

\author{
Gunjan Gupta* and Robert Van Zyl \\ Department of Electrical, \\ Electronics and Computer \\ Engineering, French South African \\ Institute of Technology, Cape \\ Peninsula University of Technology, \\ Cape Town, South Africa. \\ `E-mail: guptag@cput.ac.za \\ This paper was edited by \\ Ashutosh Sharma.
}

Received for publication November 15, 2020.

\begin{abstract}
LoRa technology is derived from chirp spread spectrum (CSS) having embedded forward error correction (FEC). A wide band is used for transmissions to counter interference and to handle frequency offsets. The paper investigates low power wide area networks (LPWAN) transmissions in the uplink, where the end nodes are powered by using energy harvested from the surroundings. Long-range (LoRa) networks demonstrate their capability to support Internet of Things (IOT) applications, where the end nodes utilize the harvested energy for transmission to gateways using different spreading factor $(S F)$ codes. The work fairly improves the throughput of the LoRa nodes while keeping the other parameters, like time duration of the energy harvesting $(E H), S F$, and transmit power, optimally. Initially, a mathematical expression is derived for collisions between packets of the end nodes; keeping this as an important factor, an algorithm is proposed that fairly assigns SFS to the nodes. Simulation results confirm the improvement in packet error rate and time on air when fewer LoRa nodes are used for lower SFs, as compared to higher SFs. The number of LoRa nodes that can communicate using $S F=7$ is almost four times as compared to using $S F=11$, while maintaining a low packet error rate. Also, for $S F=7$, changing the coding rate from 1 to 4 increases time on air by around $20 \mathrm{~ms}$, while time on air increases by $1,200 \mathrm{~ms}$ for $S F=12$. The energy efficiency is also compared for different SFS and different transmission powers. A lower SF and lower transmission powers are more suitable for smaller distance and provides better energy efficiency.
\end{abstract}

\section{Keywords}

LoRa, IoT, Energy harvesting, LoRaWAN, ToA, Gateway, Smart sensors.
The evolution of the Internet of Things (IOT) has taken the idea of connectivity to a very different level due to the rapid development of the platforms that will connect billions of the devices (Bor et al., 2016). With the merger of pervasive sensing along with remote power computations on these platforms, it is possible to collect and process data of numerous applications related to smart cities, agriculture, healthcare, and logistics. The utilization in numerous applications increases proportionally to the requirement on the network side (Gubbi et al., 2013). The network infrastructure, which services these loT applications, must be able to provide services that these applications demand as they might have different latency requirements, mobility levels, and reliability. Also, security should address the demand for different levels of mobility, latency tolerance, security, and reliability. Another important factor that affects demand is 
the range of communication: long and short. It is impossible for a single network architecture to address these diverse demands. The demands are divided among the different types of service providers. The mobile communication operators perceive the need to adjust the systems to serve short-extent machineto-machine (M2M) communication applications. The legacy networks, which were originally deployed for voice communication, and later used for multimedia applications, serve the delay-sensitive loT applications at the expense of higher protocol overhead. However, energy efficiency improvement of connected devices is one of the major challenges to be addressed (Dhillon et al., 2017). The development of a low power wide area network (LPWAN) standard also uses narrow band loT, termed LTE-2M, which enhances the performance of the existing mobile network to provide wide range coverage to loT devices (Adhikary et al., 2016; Balyan and Groenewald, 2016; Balyan and Saini, 2011; Pana et al., 2018).

An overview of LPWAN techniques is given in the studies of Petäjäjärvi et al. (2017) and Raza et al. (2017). A solution given for LPWAN is the LoRa ${ }^{\text {TM }}$ platform, which enables lower power and lower cost end devices, with a reliable backup system. The end-users (EU) communicates in sub-GHz bands (different in every country) and uses frequency shift keying (FSK) modulation, or a chirp spread spectrum (CSS) modulation, in which the signal is spread over a wide band channel, with the ability to recover quickly from noise and interference. The research community is attracted towards LoRaTM to address complex scenarios of loT. The work in the study of Georgiou and Raza (2017) questioned scalability together with the number of connected devices. The collisions between the EUs transmitting at the same time with the same SF have been analyzed. The results indicate that more gateways can be deployed in more crowded areas. The propagation range of links between EUs and the gateway is analyzed in the study of Petäjäjärvi et al. (2017), and a real network that is ideal for small area or town is deployed for analysis. The channel attenuation model is also valid for propagation near water surfaces. The results in the study of Petäjäjärvi et al. (2017) help in network deployment of base stations with respect to density of the EUs.

The applications, which are delay-sensitive and use lower data rates, utilize LoRa ${ }^{\top M}$ to provide a promising solution. The choice of SF with respect to the range of communication is an important tradeoff. In addition, no research has been conducted for energy efficiency and the device's lifespan using $\mathrm{LORa}^{\mathrm{TM}}$ in an LPWAN. To perceive the significance of these performance parameters and trade-offs, in Ireland implementations are being tested in real scenarios using test-beds (Costa et al., 2017).

The work reported in the studies of Centenaro et al. (2016), Goursaud and Gorce (2015), Vangelista et al. (2015) explains LoRa briefly; the main focus is on physical layer (PHY) and applications, with little attention paid to medium access control (MAC) protocol. Using a lower number of devices in a scenario, a testbed and its simulation results are presented in the study of Augustin et al. (2016). A traditional protocol similar to ALOHA is presented in the study of Adelantado et al. (2016) to assess the performance of LoRaWAN in a scenario with a higher number of devices, the work is not using any testbed or simulation for validation.

The way by which LoRa nodes communicate with one another, together with a reduction in energy consumption or using energy harvesting for LoRa nodes, will result in the development of sustainable and strong loT in future. The related work presented in the next section, reviews work that has already been reported in the literature. The main contributions of this paper are as follows:

1. The resource allocation used maximizes the LoRa user rates, and the LoRa users harvest energy from external sources.

2. The total time taken, including harvesting, transmission, and reception time at the gateway, is used for avoiding the collisions between transmissions between LoRa nodes.

3. A priority LoRa algorithm is proposed, which assigns specific SF to nodes having priority over other nodes.

4. LoRa technology scalability is also analyzed.

\section{Related work}

\section{Wireless sensor networks and LoRa}

A wireless sensor network (WSN) is comprised of sensors that are connected wirelessly. The performance of the network depends upon its nodes' capability to sense, process, and communicate with the destination sensor node. This depends upon two factors; how it is routed and the energy used for transmission (Gupta et al., 2020; Tanwar et al., 2014, 2019). LoRaWAN is gaining astounding equal ground in industry and small businesses. As of late, it has pulled incomparable degrees of consideration from the scholastic and exploration network. In the studies of Petäjäjärvi et al. (2017), Augustin et al. (2016), Reynders 
et al. (2016, 2017), an overview of the performance and detailed analysis of its operational requirements is given, aimed at scalability with respect to the simple ALOHA access techniques. The work done in the studies of Petäjäjärvi et al. (2017) and Georgiou and Raza (2017) is focused on end-user distance from the gateway using the highest data rate and ensuring correct demodulation. The work in the study of Adelantado et al. (2016) assumes a distribution of all end-users that ensures maximum coverage distance using the highest SF. In the study of Gupta et al. (2020), the proposed work provides new resource allocation, which enhances LoRa's capacity and improves its performance. A small cell network with a small radius is considered in the studies of Augustin et al. (2016) and (Bandopadhaya et al., 2020) such that all endusers can communicate with the gateway directly using available SFs. A three layer loT architecture is employed in the study of Bandopadhaya et al. (2020) for healthcare monitoring of soldiers working in adverse environmental conditions. The integration of NOMA with LoRa is also getting attention for resource allocation (Balyan, 2020; Balyan and Daniels, 2020; Bandopadhaya et al., 2020; Li et al., 2019). The work in the study of Sherazi et al. (2020) proposed a model to evaluate the energy consumption and predict the LoRaWAN monitoring devices' battery life. In the study of Delgado et al. (2020), the viability of LoRaWAN battery-less Class A devices is considered for both downlink and uplink transmissions. A model using a Markov chain is proposed, which uses granularity parameters and verified after comparing with a simulation model. It also checks applications-specific viability of battery-less LoRaWAN devices. The effect of the transmission interval and packet size is also analyzed, which concludes that the performance of the downlink is influenced when, especially, the second reception window is open. This work also proposes to use small size packets. The work in the study of Farooq (2020) proposes a multi-hop communication scheme for the uplink, which uses LoRa's PHY layer parameters to extend the network's coverage and throughput simultaneously.

\section{Energy harvesting and scheduling}

The requirements of high-speed networking in all the sectors is putting a burden in the form of resources to store and means to conserve energy, which is further growing due to massive sizes (Khargharia et al., 2007). The LoRa flexibility is limited when the devices are powered by energy sources (batteries). The deployment of such devices further limits the performance of the LoRa, as the battery replacement cost and distance of location or dangerous environment is another factor which needs to be considered. This clearly indicates that addressing energy efficiency is not sufficient. Another solution is to use energy harvesting, which is used to provide power to remote sensors or LoRa nodes. The harvested energy can be taken from solar energy, radio frequency energy, electromagnetic energy, or wind energy (Clerckx et al., 2019). The radio frequency energy can be derived from dedicated transmitters, for example WiFi. The work reported in the study of Orfei et al. (2017) uses a battery-less LoRa wireless sensor that monitors road conditions. Mechanical vibrations are harvested electromagnetically, using energy harvester with Halbach harvesting configuration for harvesting. The work in the study of Lee et al. (2018) proposes a novel floating device that harvests thermoelectric and solar energy. The work presented in the study of Hasanloo et al. (2020) uses a system model that has a real-time periodic task set, an energy harvester, and a hybrid energy storage system (HESS). The HESS is described in two parts: instantly available charge (IAC) and instantly unavailable charge (IUC). These two parts intelligently controls the flow of charge in HESS and prolongs the lifetime of the system. Furthermore, the combination of the HESS and task scheduling leads to lifetime improvements of up to $20 \%$ provides as compare to other classical algorithms. The work in the study of Sherazi et al. (2020) uses available resources of renewable energy in a smart industry environment to highlight the importance of energy harvesting compared to the replacement cost of battery and associated damages. In our analysis of the literature review on LoRaWAN and $E H$ together, we found that the performance of LoRaWAN is limited due to:

1. The devices that are powered by battery; and

2. Use of resource allocation algorithms, which are prone to collisions.

The work is done in the paper to address above mentioned issues. The remainder of the paper is organized as follows; an overview of LoRa specifications are given in the third section, energy harvesting and collision detection methods for LoRa nodes is explained in the fourth section, simulation results for performance evaluation are given in the fifth section, and finally, the conclusions are drawn in the sixth section.

\section{LoRa specifications}

LoRa technology is derived from chirp spread spectrum (CSS) having embedded forward error 
correction (FEC). A wide band is used for transmissions to counter interference and to handle frequency offsets. A LoRa receiver is sensitive to decoding transmissions which are $19.5 \mathrm{~dB}$ below the noise floor (Bor et al., 2016), which enables larger communication distances. The main benefits of LoRa include longrange links, robustness, low power, doppler, and multipath resistance. The available LoRa transceivers can operate between 137 and $1,020 \mathrm{MHz}$. They are used in ISM bands. The physical layer of LoRa can be used with any MAC layer; however, LoRaWAN is the MAC for LoRa using a star topology.

\section{LoRaWAN}

The LoRaWAN provisions are maintained by the LoRa alliance, which is a non-profitable organization. The devices in LoRaWAN transmit packets directly to the nearby gateway(s), denoted as GW, which transparently forward the packets to a network server (NS). The NS uses the best packet and removes multiple duplicate messages, which might arrive due to multiple gateways, and forwards the packet to the application server. The devices and application servers are supplied by the end-user (EU), while the network provider provides the gateways and network server.

The three types of end devices are defined by LoRaWAN: classes A, B, and C. Class A devices send the packet randomly to the gateway and after a waiting time opens a receive window to receive any acknowledgment or pending messages from the gateway. Class B devices work on top of Class A devices with an additional scheduled receive window. Class $C$ devices extend Class $A$ by leaving the receive window open until it is transmitting. Classes $A$ and $B$ devices are mainly battery-powered, while Class $\mathrm{C}$ devices are mains powered.

As stated earlier, LoRaWAN operates in the ISM band (license exempt band). The frequency depends upon the country of deployment and operates using on the following frequencies 433,868 or $915 \mathrm{MHz}$. There are eight physical layers used for this band; six with spreading factor ( $S F 7 \leq S F \leq 12)$ using $125 \mathrm{kHz}$ bandwidth, 1 with $S F=7$ at $250 \mathrm{kHz}$ bandwidth and the eighth operates with Gaussian frequency shift keying (GFSK) and supports $50 \mathrm{kbps}$ data rate. In order to extend the battery life of end node devices and the capacity of the network, the data rate and RF output of end nodes can be controlled independently by using an adaptive data rate (ADR) scheme. The chip rate, chip duration, bandwidth, symbol rate, symbol duration, and data rate are denoted by $R_{c}$, $T_{c}, B W, R_{s}, T_{s}$ and $R_{b}$, respectively. The notations are

given in Table 1. The relation between them are as follows:

$$
\begin{gathered}
R_{c}=B W \text { chips } / \mathrm{s} \\
T_{c}=\frac{1}{B W} \\
R_{s}=\frac{B W}{2^{S F}}=\frac{R_{c}}{2^{S F}} \text { symbols } / \mathrm{s} \\
T_{s}=\frac{2^{S F}}{B W}
\end{gathered}
$$

where $R_{c}>R_{s}$.

$$
R_{b}=S F \times \frac{B W}{2^{S F}} \times \frac{4}{4+C R}
$$

where $C R$ is the coding rate for forward error correction (FEC), $1 \leq C R \leq 4$.

With an increase in $S F, R_{b}$ decreases and vice versa. The main impact of increasing $S F$ will be on time on air (ToA), which means more time will be used to send the same amount of data. This in effect increases the power consumption too. Therefore, a higher SF value is used for a weak signal or in case of higher channel interference.

\section{Time on air}

The time on air (ToA) that not only depends upon the size of the payload, but also on the selection of $B W, S F$ and CR. It can be calculated using the Semtech LoRa modem calculator (Gubbi et al., 2013). The selection of communication parameters hugely affects the

\section{Table 1. Notations.}

\section{Notation}

$R_{0}$

$T_{c}$

$R_{s}$

$T_{s}$

$R_{b}$

$S F$

CR

$t_{a i}$

\section{Definition}
Chip rate
Chip duration
Symbol rate
Symbol duration
Data rate
Spreading factor
Coding rate
Time on air of ith node 
performance and scalability of LoRa. The ToA denoted as $t_{a_{i}}$ is given as follows:

$$
t_{a_{i}}=\frac{s}{R_{s}}=s \times T_{s}
$$

where $s$ denotes the number of symbols.

The work in this paper considers the frequency regulations on duty cycle is between 0.01 and $10 \%$ of Europe for the $868 \mathrm{MHz}$ sub-band, which is also known as ISM sub-GHz band. If the duty cycle is denoted by $T$, then $(1-T) \%$ denotes the off-time for the node, after a packet transmission, when $T=1 \%$. The $T_{i}$ is the on-time for the $i^{\text {th }}$ node during transmission, after which $T_{i}^{\text {off }}=\frac{\left(1-T_{i}\right)}{T_{i}} t_{a_{i}}$ is the off-time for the LoRa node. LoRa nodes are battery-less and uses harvested energy to power up, the harvested energy is from external sources.

\section{Energy harvesting and collision detection methods for LoRa nodes}

The model of LoRaWAN is shown in Figure 1. Each LoRa node is battery-less and is powered by harvested energy from external sources of energy. The energy can be harvested from any external source, which will not interfere with the LoRa nodes' frequencies. For a harvested energy per unit time denoted as $E_{i}$ for the $i^{\text {th }}$ node, which depends upon external conditions and is independent of time like distance from the energy sources. The transmission by a LoRa node is only possible after harvesting sufficient energy. Therefore, transmission is carried out after harvesting. Let the

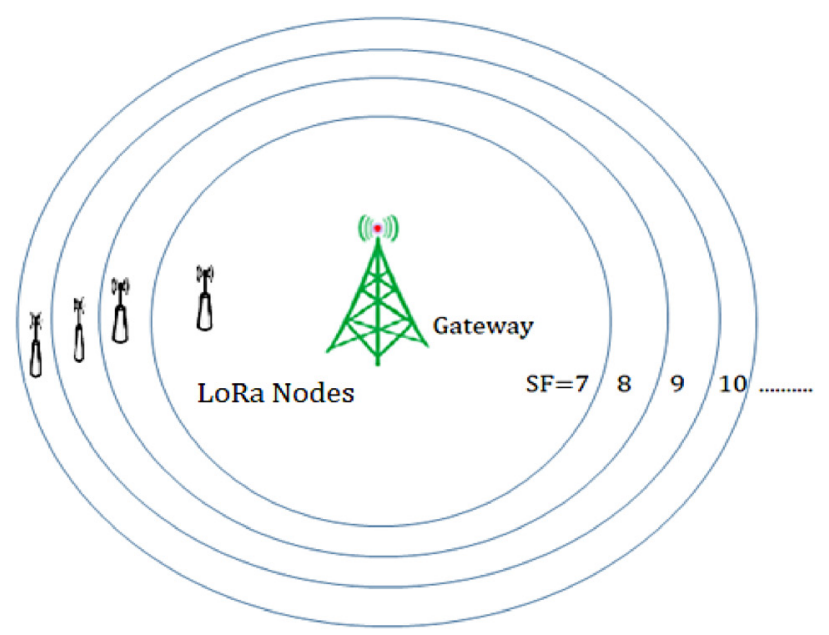

Figure 1: Model of LoRaWAN. total time taken from harvesting, transmission, and reception at the gateway for the $t^{\text {th }}$ node be:

$$
T_{i}=t_{h_{i}}+t_{a_{i}}+t_{r_{i}}
$$

where $t_{h_{i}}=T_{i}^{\text {off }}$ denotes the time of harvesting energy and $t_{r}$ denotes the time required for complete reception of the packet. Therefore, the harvested energy for the $i^{\text {th }}$ node is given by:

$$
E_{h_{i}}=t_{h_{i}} \times E_{i}
$$

Also, let the reception starts and ends at $t_{r_{i s}}$ and $t_{r_{i e}}$, respectively, for the $i^{\text {th }}$ node. The $t_{r_{i}}$ for the $i^{\text {th }}$ node is then given by:

$$
t_{r_{i}}=t_{r_{i e}}-t_{r_{i s}}
$$

The midpoint of length and midpoint of reception time $t_{r_{i}}$ is given by:

$$
t_{r_{i j}}=\frac{t_{r_{i e}}-t_{r_{i s}}}{2} \text { and } t_{r_{\text {im }}}=\frac{t_{r_{i e}}+t_{r_{i s}}}{2}
$$

The two packets $i$ and $j$ will collide during the reception time for:

$$
\left|t_{r_{i m}}-t_{r_{j m}}\right|<t_{r_{i j}}+t_{r_{j i}}
$$

The LoRa nodes are required to transmit at maximum power, denoted by $P_{\max }$, and which is known in advance. A LoRa node decides its transmission time after harvesting the energy required for transmission. If the harvested energy is more than required, it will not be stored.

A collision may occur between nodes with the same SF or different SFs. The work in this paper considers both these scenarios. Ideally, the nodes with different SFS must not interfere with each other due to orthogonality conditions; however, the imperfect orthogonality leads to inter-SF interference between these nodes.

The calculations are done taking node $i$ as reference. The collision possibilities depend upon harvesting time, time on air, and time of reception. The delay between harvesting time and transmission is considered to be zero. For any two nodes $i$ and $j$ :

- $t_{h,}$ and $t_{h,}$ denotes harvesting time.

- $T_{a_{i}}$ and $T_{a_{j}}$ denotes time on air.

- $t_{r_{i}}$ and $t_{r_{j}}$ denotes reception time.

Let the collision time between two nodes $i$ and $j$ be denoted by $t_{i, j}^{c}$. 


$$
\begin{gathered}
\text { Case1:If }\left[\left(t_{h_{i}}>t_{h_{j}}\right) \& \&\left(T_{a_{i}}>T_{a_{j}}\right) \& \&\left(t_{r_{j}}>t_{r_{j}}\right)\right] l\left(t_{h_{i}}>t_{h_{j}}+T_{a_{j}}+t_{r_{j}}\right) \\
t_{i, j}^{c}=0
\end{gathered}
$$

This case considers that the harvesting time, time on air and reception time of node $i$ is greater than that of node $j$. Also, the harvesting time of node $i$ is greater than the total time taken by another node $j$. In this case, collision time between nodes is zero, as shown in Figure 2A.

Case 2: $\left[\left(t_{h_{i}}>t_{h_{j}}\right) \& \&\left(T_{a_{i}}>T_{a_{j}}\right) \& \&\left(t_{r_{i}}>t_{r_{j}}\right)\right] \mid\left(t_{h_{i}}<t_{h_{j}}+T_{a_{j}}+t_{r_{j}}\right)$

$$
t_{i, j}^{c}=t_{h_{j}}+T_{a_{j}}+t_{r_{j}}-t_{h_{i}}
$$

This case considers that the harvesting time, time on air and reception time of node $i$ is greater than of node $j$. However, with the harvesting time of node $i$ less than the total time taken by node $j$, the collision time between nodes is given in Equation (10), as shown in Figure 2B.

Case 3: $\left[\left(t_{h_{i}}>t_{h_{j}}\right) \& \&\left(T_{a_{j}}<T_{a_{j}}\right) \& \&\left(t_{r_{i}}<t_{r_{j}}\right)\right] \mid\left(t_{h_{i}}>t_{h_{j}}+T_{a_{j}}+t_{r_{j}}\right)$

$$
t_{i, j}^{c}=0
$$

When harvesting time of node $i$ is greater than of node $j$, the time on air and reception time of node

\begin{tabular}{|c|c|c|c|c|}
\hline & $t_{h_{i}}$ & $T_{a_{i}}$ & $\overline{t_{r_{i}}}$ & \\
\hline$t_{h_{j}}$ & $T_{a_{j}}$ & & & $B$ \\
\hline
\end{tabular}
$j$ is greater than of node $i$. However, the harvesting time of node $i$ is greater than the total time taken by

\begin{tabular}{|c|c|c|c|c|}
\hline & $t_{h_{i}}$ & $T_{a_{i}}$ & $t_{r_{i}}$ & \\
\hline$t_{h_{j}}$ & $T_{a_{j}}$ & & & $D$ \\
\hline
\end{tabular}

\begin{tabular}{|l|l|l|l|}
\hline \multicolumn{2}{|c|}{$t_{h_{i}}$} & $T_{a_{i}}$ & $t_{r_{i}}$ \\
\hline$t_{h_{j}}$ & $T_{a_{j}}$ & $t_{r_{j}}$ & \\
\hline
\end{tabular}

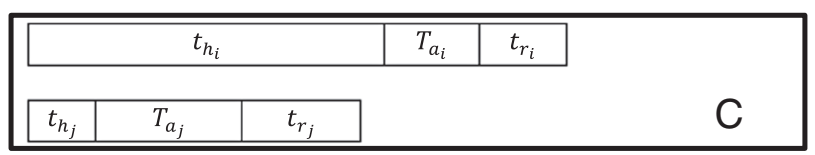

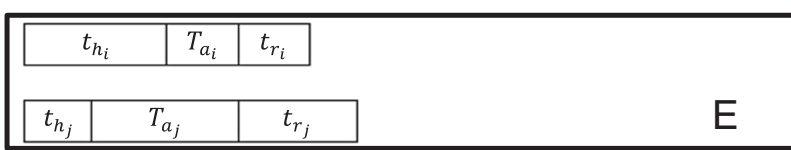

Figure 2: Illustration of collision time for nodes as a function of their harvesting time, time on air and reception time. another node $j$, then collision time between nodes is zero as shown in Figure $2 \mathrm{C}$.

$$
\begin{gathered}
\text { Case 4: }\left[\left(t_{h_{i}}>t_{h_{j}}\right) \& \&\left(T_{a_{i}}<T_{a_{j}}\right) \& \&\left(t_{i_{i}}<t_{r_{j}}\right)\right] \mid\left(t_{h_{j}}<t_{h_{j}}+T_{a_{j}}+t_{r_{j}}\right) \\
t_{i, j}^{c}=t_{h_{j}}+T_{a_{j}}+t_{r_{j}}-t_{h_{i}}
\end{gathered}
$$

When harvesting time of node $i$ is greater than of node $j$, the time on air and reception time of node $j$ is greater than of node $i$. However, the harvesting time of node $i$ is lesser than the total time taken by another node $j$, then collision time between nodes is given by Equation (12) as shown in Figure 2D.

$$
\text { Case 5: } \begin{gathered}
\left(t_{h_{i}}+T_{a_{i}}+t_{r_{i}}<t_{h_{j}}+T_{a_{j}}+t_{r_{j}}\right) \\
t_{i, j}^{c}=T_{a_{i}}+t_{r_{i}}
\end{gathered}
$$

When total time of node $i$ is lesser than the total time taken by another node $j$, then collision time between nodes is given by Equation (13) as shown in Figure 2E.

The allocation of the SF to an EU is an important decision for the LoRaWAN for many reasons, for example, a higher value of $S F$ provides higher coverage distance for better reception and suffers from lower bit rate and longer ToA. Therefore, for higher SF values the communication channels are busy for longer durations, which increases the probability of collisions between the packets transmitted concurrently. As the different SFs are orthogonal to each other, this allows more communication using the same channel. The number of devices $d_{\text {total }}$ is usually very large, the minimum date rate will do exhaustive search equal to $6^{d_{\text {total }}}$. Therefore, an exhaustive search is not used due to complexity. To reduce the complexity, the algorithm explained below is used.

The proposed PRIORLoRa algorithm assigns SF on the basis of the priority of nodes to send data. To the best of the author's knowledge, this paper is the first to assign SF to the EU with priority. Most of the algorithms in literature assign SF on the basis of radio visibility only. The algorithm is given below.

Algorithm PRIORLoRa

1. Input:

2. $d_{\text {total }}$ - number of devices covered by a gateway $S F_{s}, 7 \leq s \leq 12$ : denotes number of $S F$ of value $S$,

SENS: denotes sensitivity of the devices, RSSI - nodes power levels.

PRSSI - priority nodes power levels.

3. Output: $S F_{0}$ 
4. function PRIORLoRa-SF ([PRSSI $]_{\mathrm{m} \times \mathrm{n}},\left[S F_{1 \times n}\right)$.

5. $[S]_{1 \times n}=[7 \ldots 12]$ of $n$ end devices.

6. for $I=1$ to length $\left(S F_{s}\right)$

7. $c=\operatorname{count}(\operatorname{PRSSI}(I)>\operatorname{SENS})$

8. if $c>\frac{d_{\text {total }}}{\text { length }\left(S F_{s}\right)}$

9. $r=\frac{d_{\text {total }}}{\text { length }\left(S F_{s}\right)}$

10. else

11. $r=c$

12. End if

13. for $k=0$ to $r$

14. $[p, q]=\max (P R S S I)$

15. $S F_{o}[q]=[S F[/]]$

16. PRSSI $[:, q]=-200 \mathrm{dBm}$

17. End for

18. End for

19. return $S F_{o}$.

The complexity is reduced by searching for $r$ devices only. The complexity in searching SF for a node (user) using the algorithm is $6^{r}<6^{d_{\text {total }}}$ (the number of SF used are 6 in this algorithm). For $m$, SFs used complexity will be $m^{r}<m^{d_{\text {total }}}$. The flowchart of proposed PRIORLoRa algorithm is shown in Figure 3.

The energy consumed by LoRa node during transmission of packet is found using the current levels given in the study of Gubbi et al. (2013). This energy needs to be minimize even though energy harvesting is used for LoRa nodes. The energy spent (Joules) in transmission for $i^{\text {th }}$ node for a supply voltage $V$ volts and current I mA can be calculated from:

$$
E_{i}=V \times I \times t_{a_{i}}
$$

The energy spent during transmission plays an important role in improving the performance of the network. The energy efficiency $(E E)$ for $i^{\text {th }}$ node is defined as:

$$
E E_{i}=\frac{\text { Payload in bits } \times p_{s}}{B W \times E_{i}}
$$

where $p_{r}$ denotes the probability of packet received without error.

The number of transmitted packets by a node depends upon SF, bandwidth of channel used and the payload. If the payload is large, the packets become larger which leads to lesser transmission in

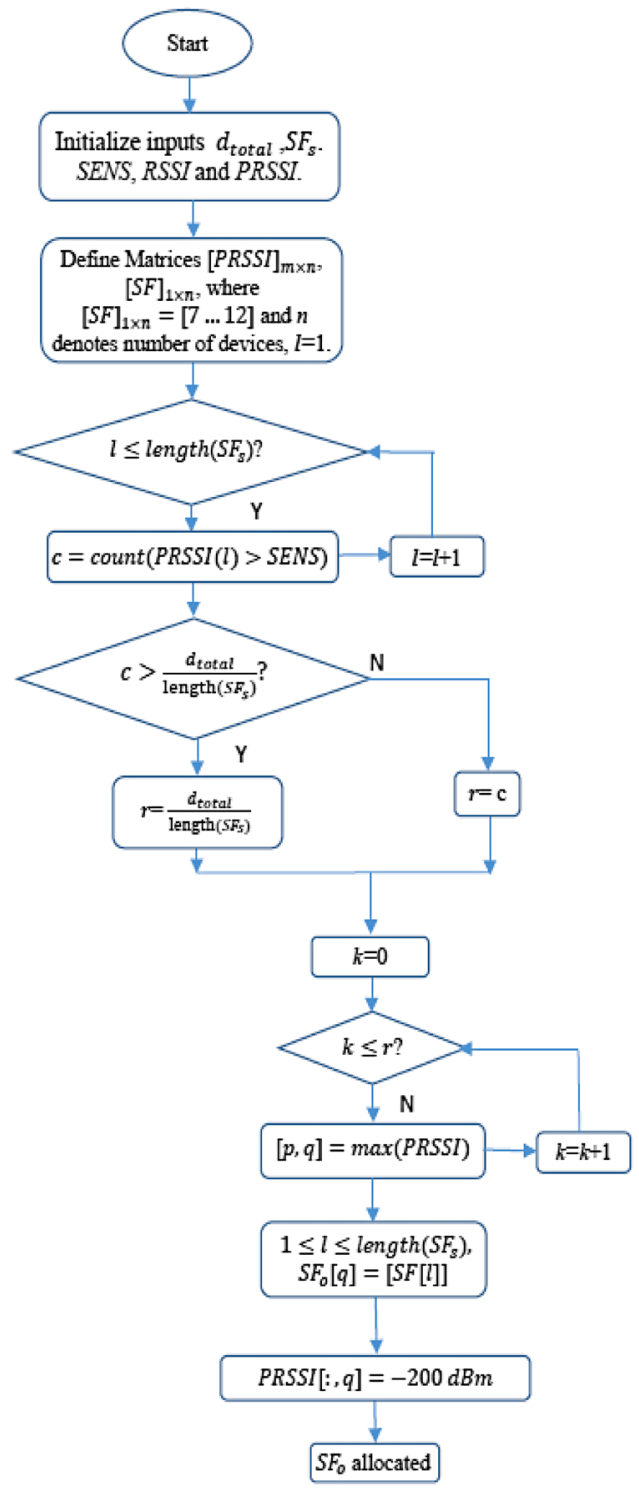

Figure 3: Flowchart of PRIORLoRa algorithm.

a day. One way to use the battery or the harvested energy of a LoRa node for a long time is utilization of longer SF, which increases time on air.

\section{Simulation results for performance evaluation}

The simulation is done in MATLAB implementing the PRIORLoRa-SF algorithm described in the previous section. A LoRa network is implemented with end-users or nodes denoted as $d_{\text {total }}$ and a single gateway centrally placed around which nodes are randomly distributed in circles as shown in Figure 1. 
The density of the nodes is Traffic $_{d}=\frac{d_{\text {total }}}{\pi R^{2}}$, where $R$ is the total radius of the network. The probability of node present at a distance $x$ from the gateway can be found using probability density function defined as $f(x)=2 \times \frac{Y}{R^{2}}, 0 \leq y \leq x$.

The $S F s$ assigned to nodes $S F_{s}, 7 \leq s \leq 12$. The index of assigned $C R$ is from 0 to 4 , which corresponds to coding rate $1,4 / 5,4 / 6,4 / 7$, and $4 / 8$, respectively. The coding rate 1 indicates that no coding is applied. The nodes have same configuration and uses same transmit power $14 \mathrm{dBm}$ which is power allowed for the exempted frequency sub bands. Each $i^{\text {th }}$ node selects a particular available frequency channel for transmission and then wait for $T_{i}^{\text {off }}$ period before starting next transmission as per LoRaWAN specifications. The simulation parameters are taken from the study of El-Aasser et al. (2019).

In Figure 4, data extraction rate (DER) also known as success probability as a function of spreading factor $(S F)$ is plotted for three different node densities, $10 \frac{\text { nodes }}{\mathrm{km}^{2}}, 20 \frac{\text { nodes }}{\mathrm{km}^{2}}$, and $500 \frac{\text { nodes }}{\mathrm{km}^{2}}$. It is clearly evident from result that as the node density and SF increase, the DER decreases significantly. The reason in sharp fall in DER when $S F \geq 10$ is longer time on air that increases number of transmission at the same time for all three density distributions. This decrease is more prominent for node density $500 \frac{\text { nodes }}{\mathrm{km}^{2}}$, due to increase in more collisions.

The average received signal strength indication $(R S S I)$ of the message received at the gateway plays an important role in deciding a specific SF which depends upon the path loss propagation model. The SF assigned to an end user play an important role as the $S F$ increases the coverage distance of end node communication with gateway improves. This comes at a cost of longer time on air and lower bit rate. Usually $10 \leq S F \leq 12$ are considered high SFS, which will have longer time on air and have higher probability of collisions. However, the use of different SF provides orthogonality in communication or in other words less interference. This leads to the inclusion of more number of nodes or higher spectral efficiency. The effect of payload of a packet is explored. The variation in packet error rate (PER) in a network that has increasing LoRa nodes and payload used are of 10, 25,40 , and 60 bytes are is done in this section. The PER is mainly affected by the number of collisions on the communication channel used by more than one node at a time. These collisions are influenced by the number of LoRa nodes that are using the same SF on the same channel.

In Figures 5 and 6, the rate of packet error is plotted against the number of LoRa nodes equal to 4,000 and 8,000, respectively, at constant $S F=7$, duty cycle $0.02 \%$, and constant coding rate $=4 / 5$. These values are close to real-time loT applications. A higher value of packet error rate indicates lesser packets are received with errors and vice versa. In Figure 5, the number of LoRa nodes used are 4,000, when the payload is 10 bytes the PER is more than

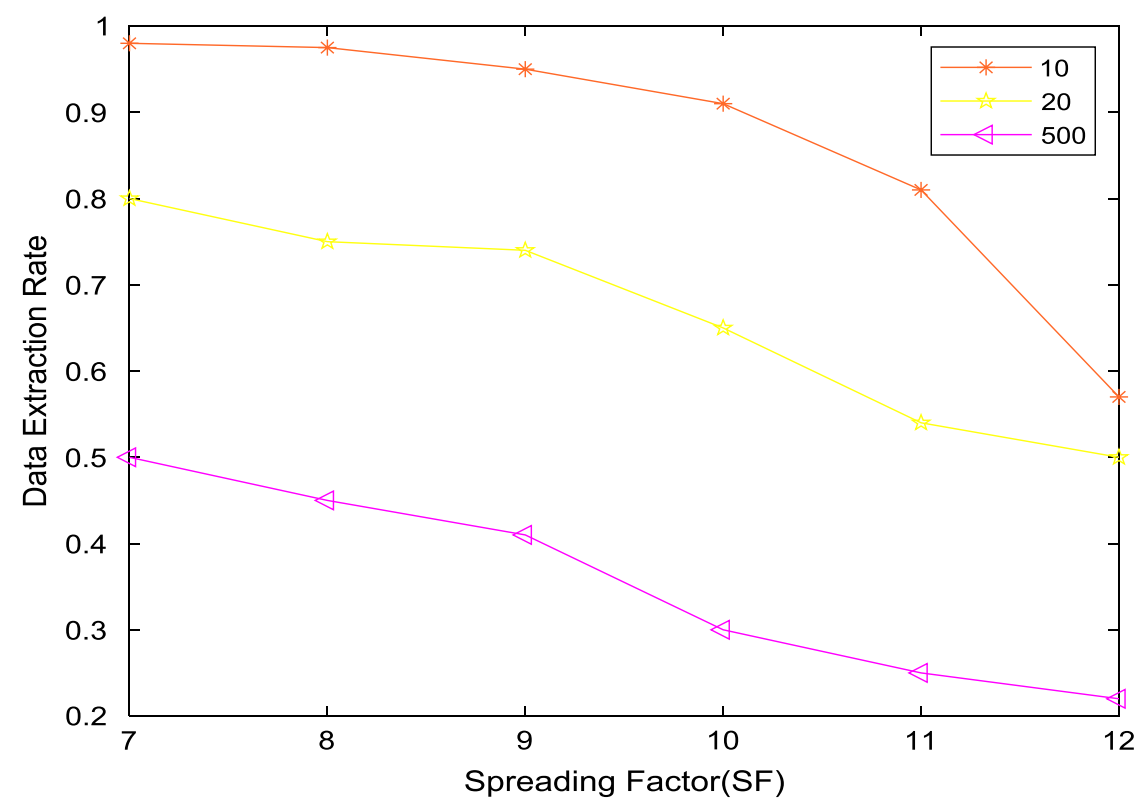

Figure 4: Data extraction rate vs spreading factor (SF) for three different densities $10 \frac{\text { nodes }}{\mathrm{km}^{2}}, 20 \frac{\text { nodes }}{\mathrm{km}^{2}}$ and $500 \frac{\text { nodes }}{\mathrm{km}^{2}}$ square $\mathrm{km}$. 


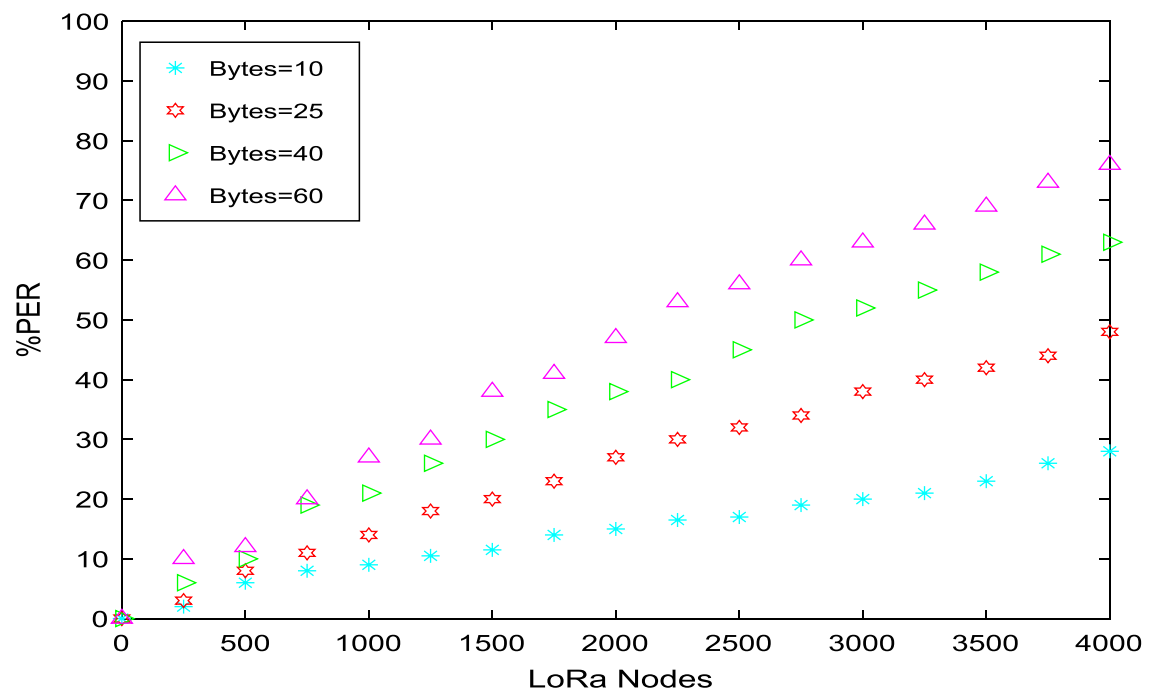

Figure 5: The rate of increase of packet error as a function of increase in number of LoRa nodes for constant $S F=7$.

$25 \%$, for an increase in the payload to 60 bytes the payload becomes almost thrice of it, i.e. close to $75 \%$. Ideally, the packet error rate must be below $10 \%$.

As the number of LoRa nodes increases the packet error rate increases drastically. Also, an increase in payload brought a higher packet error rate when fewer LoRa nodes are employed as shown in Figure 6. Clearly from Figure 6, the number of LoRa nodes that lead to a $10 \%$ packet error rate is around $2,000,1,000,500$, and 200, respectively, for 10, 25, 40 , and 60 byte payloads. At 8,000 LoRa nodes, more than $90 \%$ are received with error for a payload of 60 bytes. The higher packet error rate is due to the larger number of LoRa nodes in Figure 6.

In Figures 7 and 8, the effect on packet error rate is compared with the number of LoRa nodes. The maximum number of LoRa nodes employed are 800 and 1,800 in Figures 7 and 8, respectively, keeping constant $S F=11$. An increase in $S F$ leads to a very high packet error rate due to a longer time on air. For a payload of 60 bytes, the packet error rate is around $97 \%$ and more than $100 \%$ for 800 and 1,800

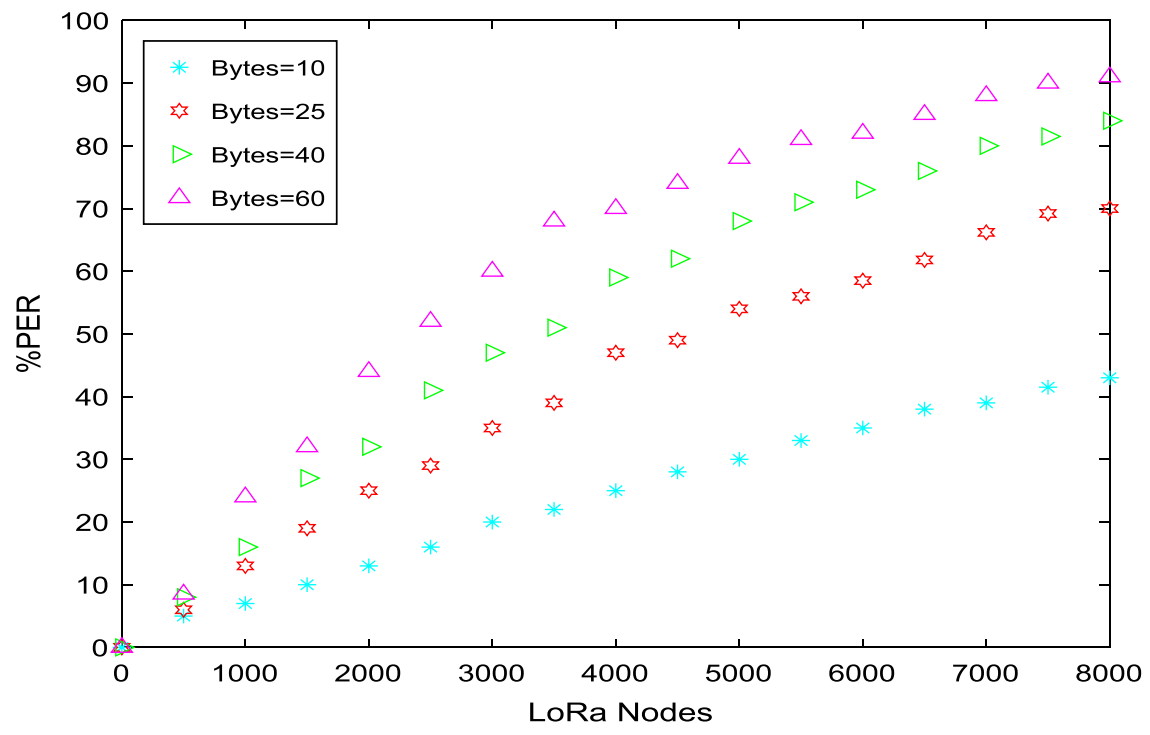

Figure 6: The rate of increase of packet error as a function of increase in number of LoRa nodes for constant $S F=7$. 


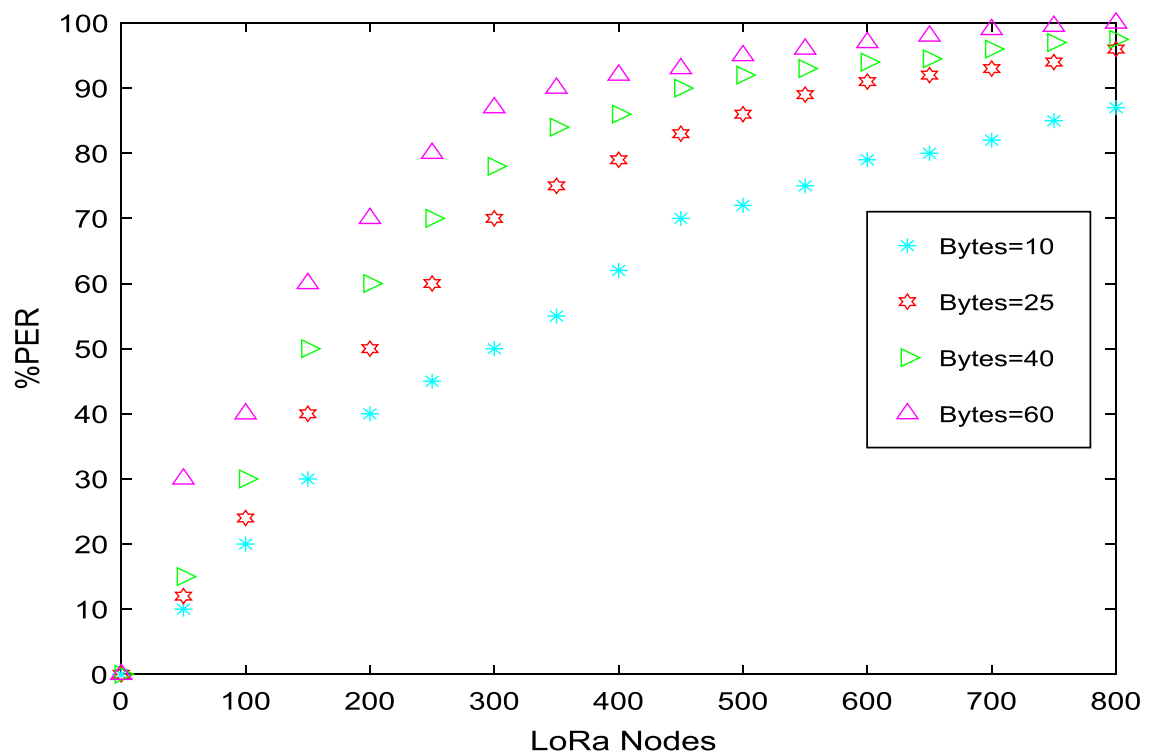

Figure 7: The rate of increase of packet error as a function of increase in number of LoRa nodes for constant $S F=11$.

LoRa nodes, respectively. Compared to the results of Figures 5 and 6 , where $S F=7$ is used for a payload of 60 bytes the packet error rate is around 75 and 92\%, respectively, for 4,000 and 8,000 LoRa nodes. It is due to the higher time on air which is due to more collisions affected by the payload of packets and SF.

In Figure 9, the packet error rate is compared for two different duty cycles 0.2 and $0.5 \%$, while keeping SF constant at 7. A higher duty cycle means longer time on air, the effect of which is evident from the plot of Figure 9, that packet error rate reaches 100\% for around 600 LoRa nodes for $0.5 \%$ duty cycle as compared to $82 \%$ packet error rate for 800 LoRa nodes when 0.2 duty cycle is employed. The result indicates the importance of selecting an appropriate duty cycle for a specific SF. It is also important to consider regulations imposed by the ISM operational band which allows a maximum of $1 \%$ duty cycle.

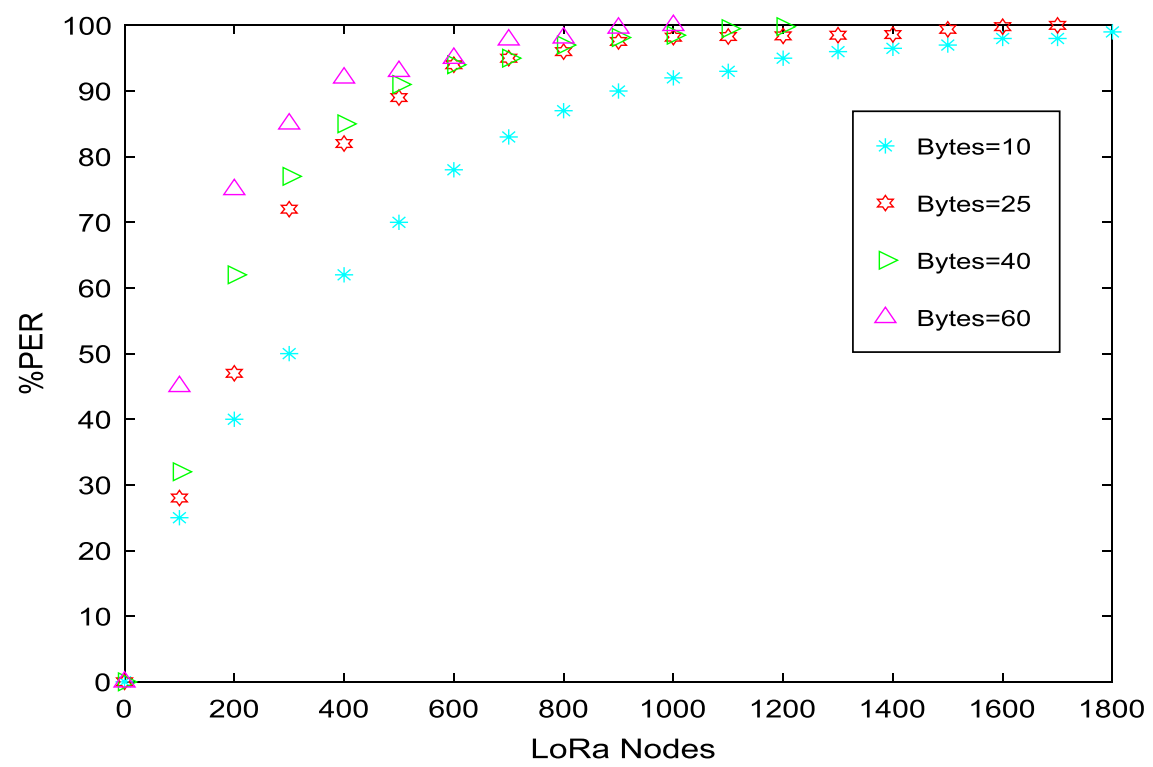

Figure 8: The rate of increase of packet error as a function of increase in number of LoRa nodes for constant $S F=11$. 


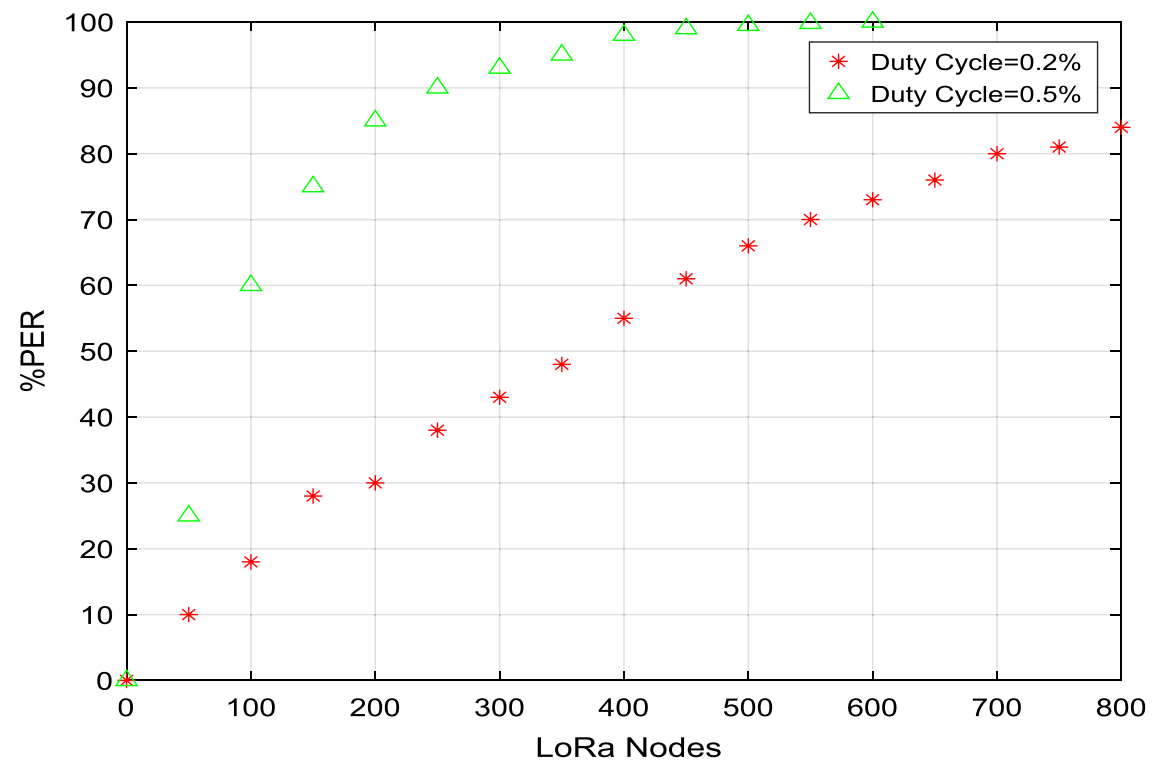

Figure 9: The rate of increase of packet error as a function of increase in number of LoRa nodes and duty cycle for constant $S F=7$.

In Figure 10, time on air is compared for different payloads using different coding rates for constant $S F=7$. The different coding rates used here are due to the different distances between LoRa nodes and gateway. The lower coding rate means a larger distance between LoRa node and gateway. The change of coding rate at constant SF improves the packet error rate as the number of encoding bits are increased. However, it is achieved at the expense of increase in the time on air.

In Figure 11, time on air is compared for different payloads using different coding rates for constant $S F=12$. From Equations (1) and (3), using time on air which is directly proportional to $2^{S F}$, increasing $S F$ from 7 to 12, for a payload of 10 bytes increases ToA to around $8,000 \mathrm{~ms}$ for $S F=12$. The use of a higher

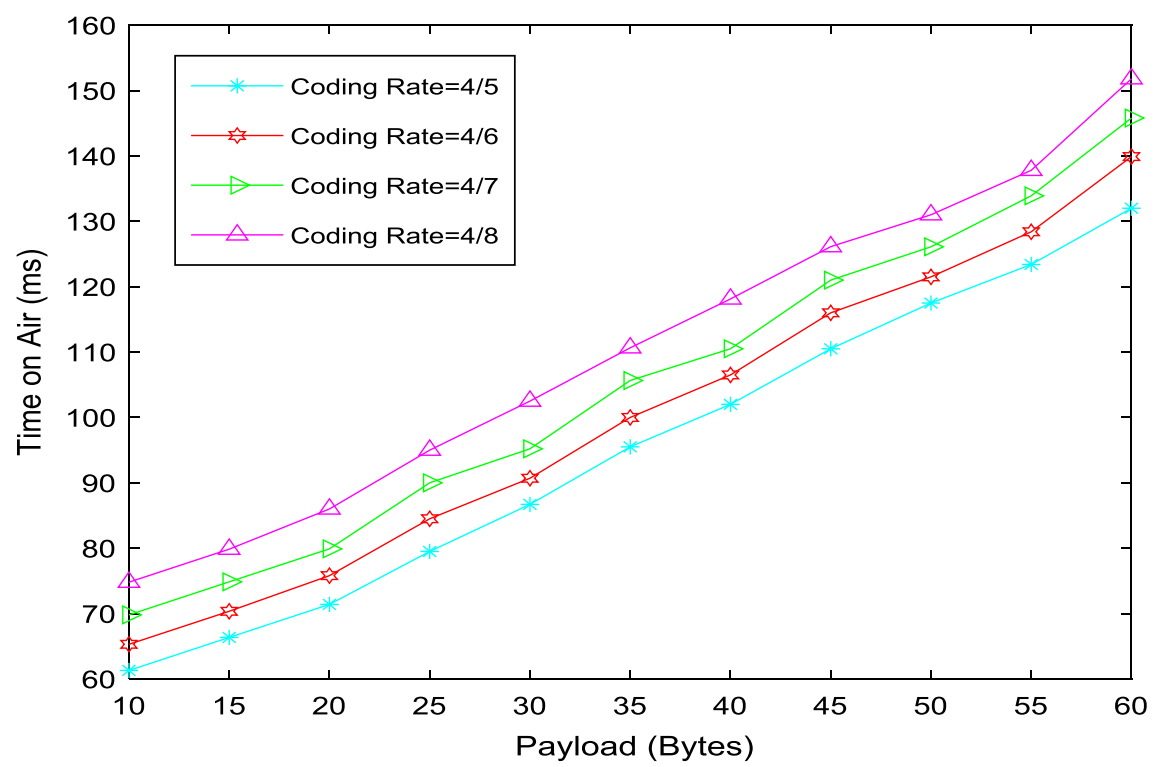

Figure 10: Time on air (ms) as a function of payload (bytes) for different $C R$ and constant $S F=7$. 


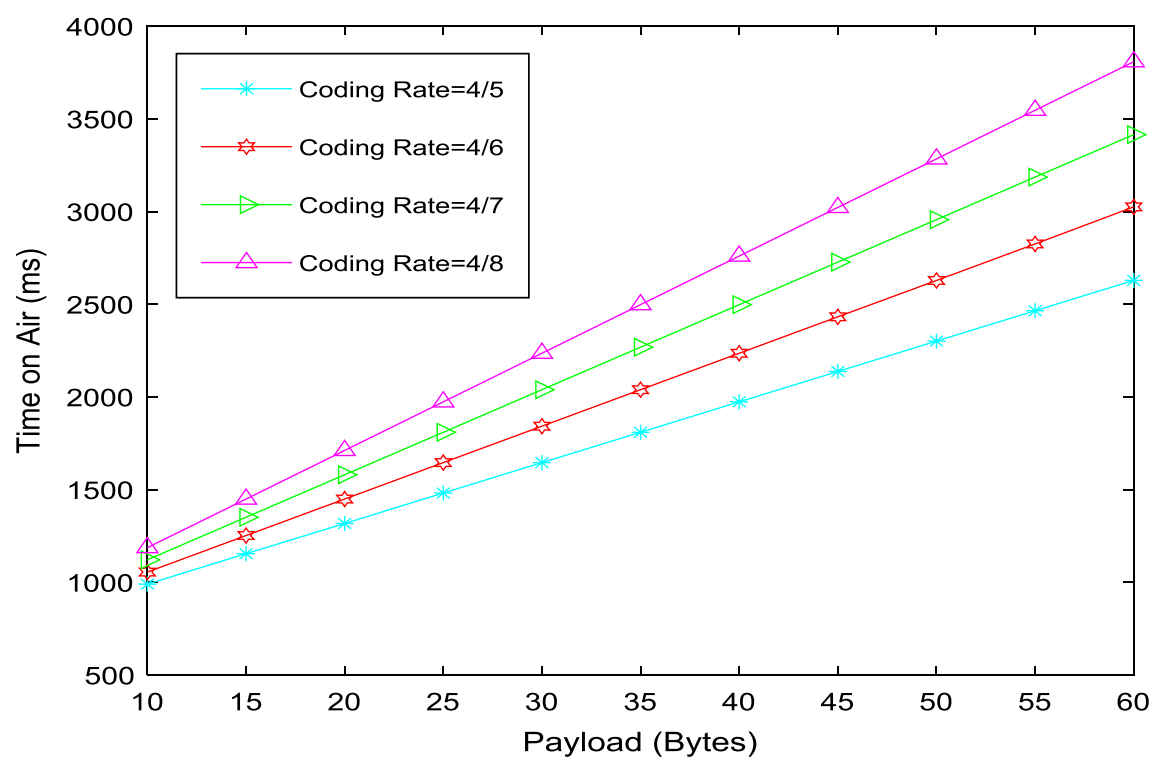

Figure 11: Time on air (ms) as a function of payload (bytes) for different $C R$ and constant $S F=12$.

coding rate makes transmission more robust. A higher coding rate must be used when the interferences present are too many.

For the illustration of the energy efficiency is expressed in bits per Hertz per Joule and the voltage used is 3.3 volts. The energy spent depends upon the current given by in [2] and is calculated using (14). A payload of 10 bytes and the bandwidth used is $125 \mathrm{kHz}$ for analysis of $E E$.

In Figure 12, the EE variation of different SFs with distance is compared. From the result, it is clear that small SFs are more suitable for small distances

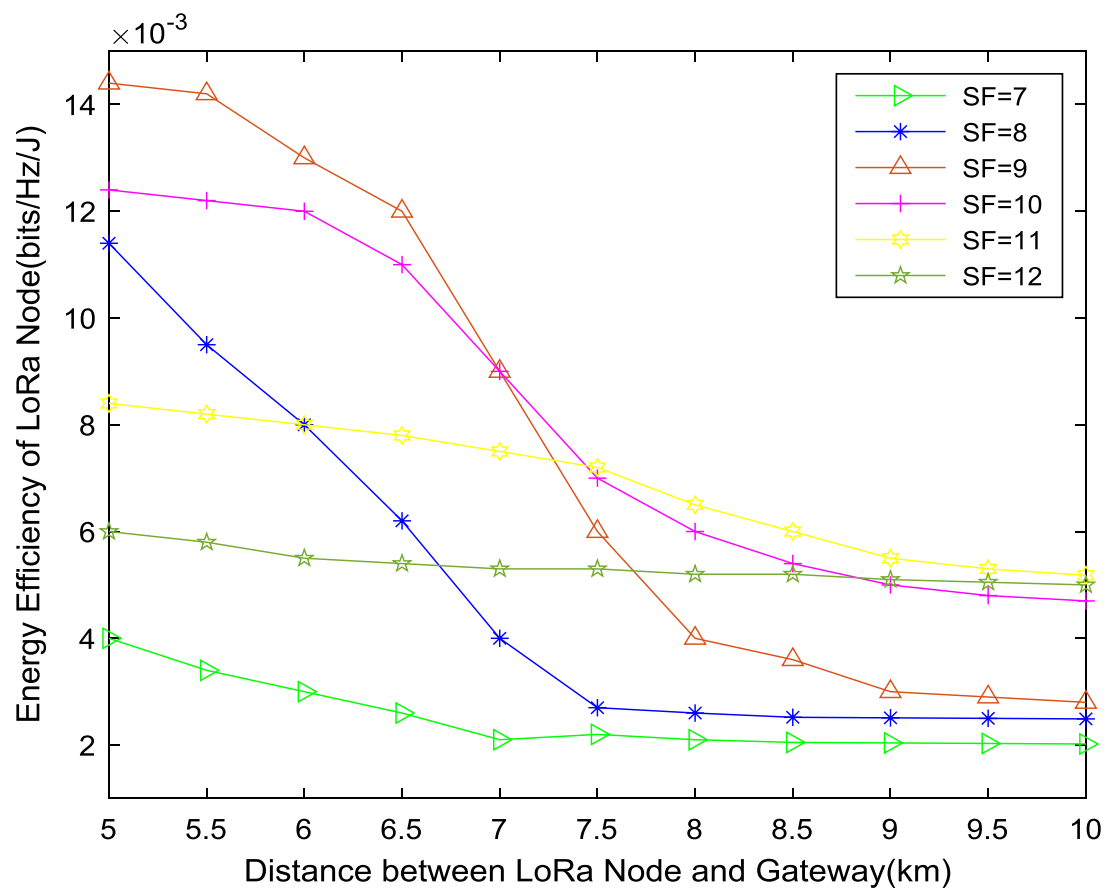

Figure 12: Comparison of energy efficiency of LoRa node versus distance between LoRa node and gateway for different $S F$. 


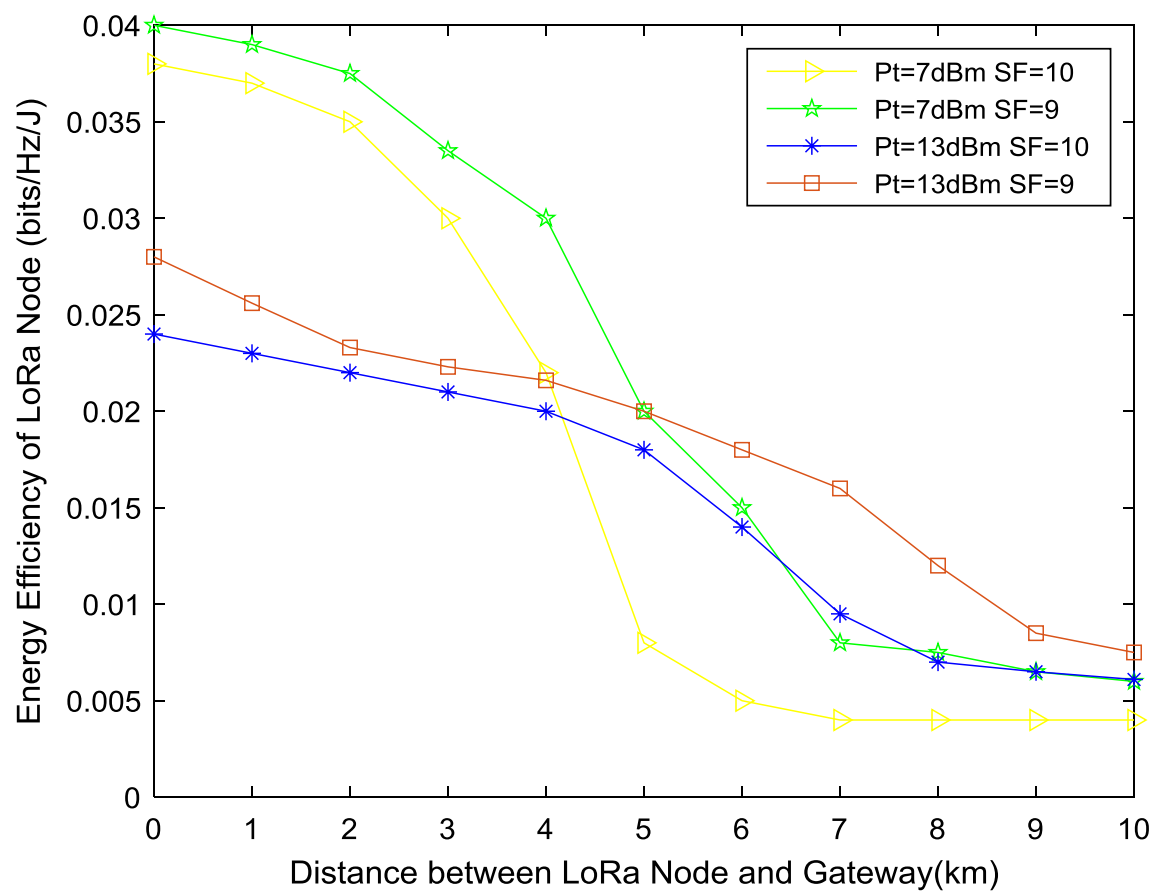

Figure 13: Comparison of energy efficiency of LoRa node versus distance between LoRa node and gateway for $S F=9$ and 10 with different transmission powers.

5-7 km, but their EE decreases due to higher number of transmissions with reduced probability of success. The higher SF provides better EE as compared to lower $S F$ values due to longer time in air. The EE of $S F=12$ is best, as its $E E$ is almost a straight line due to longer time on air for all distances.

In Figure 13, the EE variation using two transmission powers 7 and $13 \mathrm{dBm}$ with distance are compared for $S F=9$ and $S F=10$. When the transmission power is less it is more suitable for smaller distances and $E E$ is higher, however reducing $S F$ from 10 to 9 increases EE slightly for both smaller and larger distances. When the transmission power increased to $13 \mathrm{dBm}$, for both $S F=9$ and $S F=10$ the $E E$ for performance is more suitable for small and mid-range distances.

The LoRa network can ensure the best performance when the number of retransmissions is minimum, which are directly proportional to the number of collisions. This can be achieved by keeping the packet error rate below $10 \%$. One way to ensure this is by assigning SF to LoRa nodes depending upon their distance from the gateway. As the distance from the gateway increases, the SF assigned to a node increases. This will reduce the number of collisions.

Another way to ensure minimum retransmission is that the LoRa nodes must use the nearest gateway for communication, which increases the probability of having a fewer number of nodes at larger distances. This in turn limits the number of nodes with higher $S F$. This also increases the existence of orthogonality between different LoRa nodes using the same channel but with different SF. The FEC can also be adopted to reduce the number of retransmissions also.

\section{Conclusion}

In this paper, the performance analysis of the LoRa technology is done which can be achieved by performing a scalability analysis. Thus, the main motivation was to determine the number of LoRa nodes that can be supported in an loT like network. In other words, the scalability analysis is carried out, using different payloads, spreading factors, duty cycle, and coding rates. A packet is used for the transfer of information, a packet with error or collisions changes the performance of the network significantly. The packet error rate increases with an increase in payloads, duty cycle, spreading factors, and coding rate. A priority LoRa algorithm is proposed which provides priority to time-sensitive applications and ensures the allocation of $S F$ is not only on the basis of radio visibility. The work also uses energy harvesting for LoRa nodes which are battery-less. This study is useful in choosing and configuring parameters of LoRa/LoRaWAN. The parameters that are varied for 
analysis are $S F$, coding rate, network densities per square $\mathrm{km}$, duty cycle, and payload. As the number LoRa nodes which can use the same channel using spreading factor 7 for communication purposes are around 1,300 for a payload of 10 bytes. The SF and coding rate must be on the basis of the distance of the LoRa node from the gateway. The EE of LoRa node is compared for different SFs and transmission power, the results indicate that higher power and higher SF are more suitable for larger distances.

In the future, work can be done to evaluate the performance under realistic conditions using different propagation models. Also, the effect of multi-user interference can be evaluated for better scalability analysis.

\section{Literature Cited}

Adelantado, F., et al. 2016. "Understanding the limits of LoRaWAN", Proceedings of the 2016 International Conference on Embedded Wireless Systems and Networks, Austria.

Adhikary, A., Lin, X. and Eric Wang, Y. P. 2016. Performance evaluation of NB-IoT coverage. IEEE Vehicular Technology Conference, Montreal, QC, doi: 10.1109/VTCFall.2016.7881160.

Augustin, A., et al. 2016. "A study of Lora: long range \& low power networks for the internet of things", Sensors (Switzerland) 16(1466): 1-8. doi: 10.3390/s16091466.

Balyan, V. 2020. Outage probability of cognitive radio network utilizing non orthogonal multiple access. 2020 7th International Conference on Signal Processing and Integrated Networks, SPIN 2020, pp. 751-755, doi: 10.1109/SPIN48934.2020.9071401.

Balyan, V. and Daniels, R. 2020. Resource allocation for NOMA based networks using relays : cell centre and cell edge users. International Journal On Smart Sensing and Intelligent Systems 13(1): 1-18, doi: 10.21307/ijssis-2020-031.

Balyan, V. and Groenewald, B. 2016. UMTS and LTE interfaces utilization improvement with QoS in mobile communication systems. 2016 International Conference on Recent Advances and Innovations in Engineering, ICRAIE 2016, Jaipur, doi: 10.1109/ICRAIE.2016.7939466.

Balyan, V. and Saini, D. S. 2011. Integrating new calls and performance improvement in OVSF based CDMA networks. International Journal of Computer and Communications 5(2): 35-42.

Bandopadhaya, S., Dey, R. and Suhag, A. 2020. Integrated healthcare monitoring solutions for soldier using the internet of things with distributed computing. Sustainable Computing: Informatics and Systems 26(2020): 100378, doi: 10.1016/j.suscom.2020.100378.

Bor, M., et al. 2016. Do LoRa low-power wide-area networks scale?. MSWiM 2016 - Proceedings of the 19th
ACM International Conference on Modeling, Analysis and Simulation of Wireless and Mobile Systems, doi: 10.1145/2988287.2989163.

Centenaro, M., et al. 2016. Long-range communications in unlicensed bands: The rising stars in the IOT and smart city scenarios. IEEE Wireless Communications 23(5): 60-67, doi: 10.1109/MWC.2016.7721743.

Clerckx, B., et al. 2019. Fundamentals of wireless information and power transfer: From RF energy harvester models to signal and system designs. IEEE Journal on Selected Areas in Communications 37(1): 4-33, doi: 10.1109/JSAC.2018.2872615.

Costa, M., Farrell, T. and Doyle, L. 2017. On energy efficiency and lifetime in low power wide area network for the Internet of Things. 2017 IEEE Conference on Standards for Communications and Networking, CSCN 2017, Helsinki, doi: 10.1109/CSCN.2017.8088631.

Delgado, C., et al. 2020. Battery-less LoRaWAN communications using energy harvesting: modeling and characterization. IEEE Internet of Things Journal 4662(c): 1-1, doi: 10.1109/jiot.2020.3019140.

Dhillon, H. S., Huang, H. and Viswanathan, H. 2017. Wide-area wireless communication challenges for the Internet of Things. IEEE Communications Magazine, doi: 10.1109/MCOM.2017.1500269CM.

El-Aasser, M., Elshabrawy, T. and Ashour, M. 2019. Joint spreading factor and coding rate assignment in LoRaWAN networks. 2018 IEEE Global Conference on Internet of Things, GCloT 2018, Alexandria, doi: 10.1109/GCloT.2018.8620147.

Farooq, M. O. 2020. Clustering-based layering approach for uplink multi-hop communication in LoRa networks. IEEE Networking Letters 2(3): 132-135, doi: 10.1109/Inet.2020.3003161.

Georgiou, O. and Raza, U. 2017. Low power wide area network analysis: can LoRa scale?. IEEE Wireless Communications Letters 6(2): 162-165, doi: 10.1109/ LWC.2016.2647247.

Goursaud, C. and Gorce, J. M. 2015. Dedicated networks for IOT: PHY/MAC state of the art and challenges. EAl Endorsed Transactions on Internet of Things 1(1): 150597, doi: 10.4108/eai.26-10-2015.150597.

Gubbi, J., et al. 2013. Internet of Things (IoT): a vision, architectural elements, and future directions. Future Generation Computer Systems 29(7): 1645-1660, doi: 10.1016/j.future.2013.01.010.

Gupta, S. K., et al. 2020. Energy efficient routing protocols for wireless sensor network. Advances in Intelligent Systems and Computing, 275-298, doi: 10.1007/978-3-030-40305-8_14.

Hasanloo, M., Kargahi, M. and Jalilian, S. 2020. Dynamic harvesting- and energy-aware real-time task scheduling. Sustainable Computing: Informatics and Systems 28: 100413, doi: 10.1016/j.suscom.2020.100413.

Khargharia, B., et al. 2007. Autonomic power \& performance management for large-scale data centers. Proceedings - 21st International Parallel and Distributed 
Processing Symposium, IPDPS 2007; Abstracts and CDROM, Long Beach, CA, doi: 10.1109/IPDPS.2007.370510.

Lee, W. K. et al. 2018. Multi-source energy harvesting and storage for floating wireless sensor network nodes with long range communication capability. IEEE Transactions on Industry Applications, 54(3), doi: 10.1109/ TIA.2018.2799158.

Li, K., Benkhelifa, F. and McCann, J. 2019. Resource allocation for non-orthogonal multiple access (NOMA) enabled LPWA networks. 2019 IEEE Global Communications Conference, GLOBECOM 2019 - Proceedings, Waikoloa, HI, doi: 10.1109/GLOBECOM38437.2019.9013575.

Orfei, F., Benedetta Mezzetti, C. and Cottone, F. 2017. Vibrations powered LoRa sensor: an electromechanical energy harvester working on a real bridge. Proceedings of IEEE Sensors, Orlando, FL, doi: 10.1109/ ICSENS.2016.7808752.

Pana, V., Balyan, V. and Groenewald, B. 2018. Fair allocation of resources on modulation and coding scheme in LTE networks with carrier aggregation. Proceedings - IEEE 2018 International Conference on Advances in Computing, Communication Control and Networking, ICACCCN 2018, Greater Noida, doi: 10.1109/ ICACCCN.2018.8748355.

Petäjäjärvi, J., et al. 2017. Performance of a low-power wide-area network based on LoRa technology: Doppler robustness, scalability, and coverage. International Journal of Distributed Sensor Networks 13(3): 1-16. doi: 10.1177/1550147717699412.

Raza, U., Kulkarni, P. and Sooriyabandara, M. 2017. Low power wide area networks: an overview. IEEE
Communications Surveys and Tutorials 99: 1-19, doi: 10.1109/COMST.2017.2652320.

Reynders, B., Meert, W. and Pollin, S. 2016. Range and coexistence analysis of long range unlicensed communication. 2016 23rd International Conference on Telecommunications, ICT 2016, Thessaloniki, doi: 10.1109/ICT.2016.7500415.

Reynders, B., Meert, W. and Pollin, S. 2017. Power and spreading factor control in low power wide area networks. IEEE International Conference on Communications, Paris, doi: 10.1109/ICC.2017.7996380.

Sherazi, H. H. R., et al. 2020. Energy-efficient LoRaWAN for Industry 4.0 applications. IEEE Transactions on Industrial Informatics $X(X)$ : 1-1, doi: 10.1109/ tii.2020.2984549.

Tanwar, S., Kumar, N. and Niu, J. W. 2014. EEMHR: energy-efficient multilevel heterogeneous routing protocol for wireless sensor networks. International Journal of Communication Systems 27(9): 1289-1318, doi: 10.1002/ dac. 2780 .

Tanwar, S., Verma, S. and Tyagi, S. 2019. Energy harvesting-based two-hop clustering for wireless mesh network. Advances in Intelligent Systems and Computing, Springer, pp. 463-473, doi: 10.1007/978981-13-1708-8_42.

Vangelista, L., Zanella, A. and Zorzi, M. 2015. Longrange loT technologies: the dawn of LoRaTM. Lecture Notes of the Institute for Computer Sciences, SocialInformatics and Telecommunications Engineering, LNICST, Ohrid, Macedonia, doi: 10.1007/978-3-31927072-2_7. 